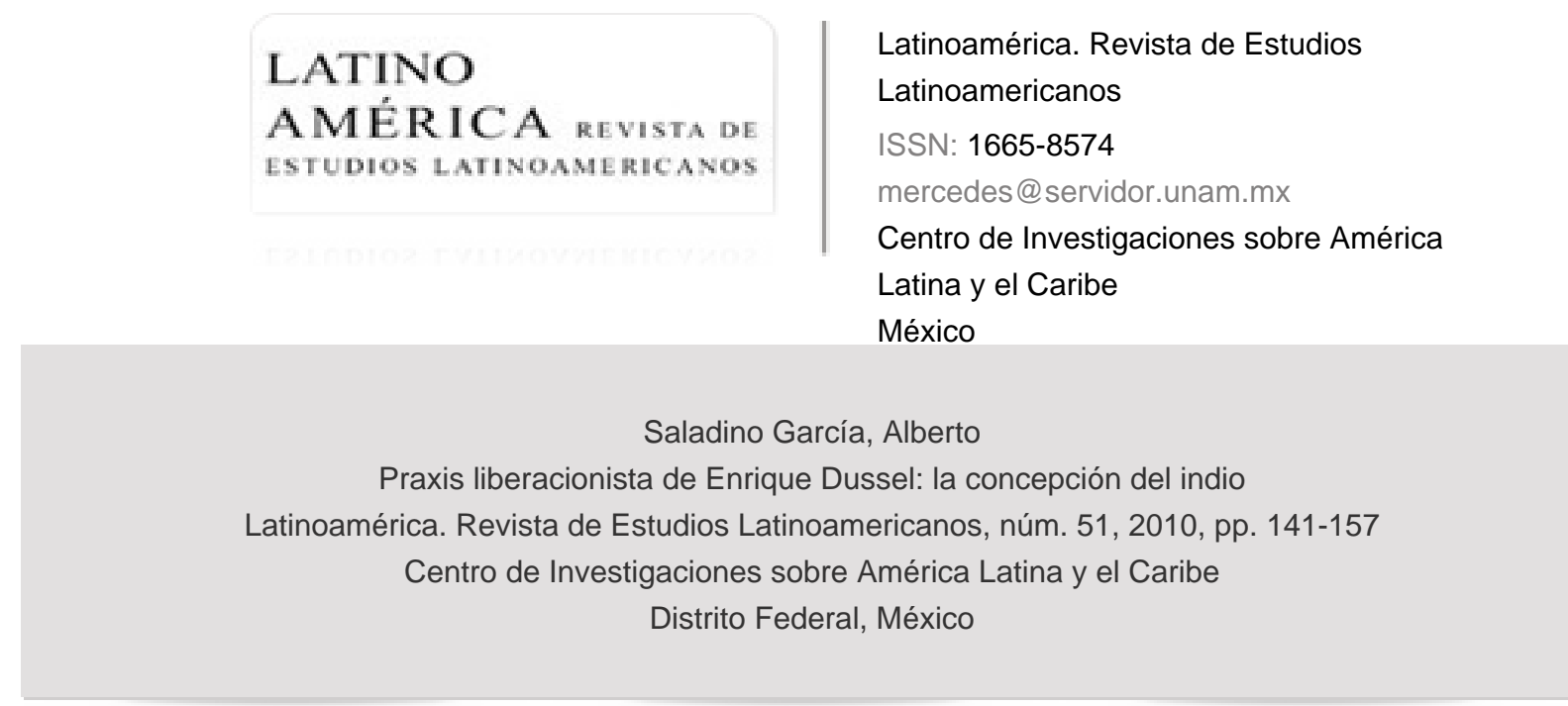

Disponible en: http://www.redalyc.org/articulo.oa?id=64015153007

- Cómo citar el artículo

- Número completo

- Más información del artículo

- Página de la revista en redalyc.org

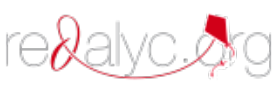

Sistema de Información Científica

Red de Revistas Científicas de América Latina, el Caribe, España y Portugal Proyecto académico sin fines de lucro, desarrollado bajo la iniciativa de acceso abierto 


\title{
Praxis liberacionista de Enrique Dussel: la concepción del indio
}

\author{
Alberto Saladino García*
}

Resumen: Enrique Dussel es uno de los foriadores de la filosofía de la liberación cuya impronta estriba en desplegar una crítica radical de carácter anticapitalista. Toda vez que en su amplia y rica obra existen diversas referencias al indio americano, me pareció pertinente sistematizar sus reflexiones al respecto. Lo corroboran dos de sus obras: su tesis doctoral en la década de los años sesenta y otro texto de los años noventa, que apareció con dos tífulos diferentes. En sus planteamientos se bordan tópicos para construir la explicación de la génesis del problema del indio latinoamericano, su ubicación como colonizado y las posibilidades acerca de la necesaria superación de esa situación.

Palabras clave: América Latina, Filosofía de la liberación, Indio, Praxis.

ABSTRACT: Enrique Dussel is one of the forgers of the philosophy of liberation whose imprint is to deploy a radical anti-capitalist critique. Given that in his broad and rich work are several references to aboriginals in America, it seemed to me appropriate to systematize his thoughts on that matter. Two works so establish: his doctoral thesis in the decade of the sixties and other text in the decade of the nineties, which appeared with two different titles. In their approach are embroidered topics to build an explanation of the genesis of the problem of the indigenous people in Latin-America, their status as colonized and possibilities about the necessary improvement of that situation.

KEY words: Latin-America, Philosophy of Liberation, Indian, Praxis.

"Facultad de Humanidades, uaEm (asaladinog@uaemex.mx). 


\section{PRESENTACIÓN}

ursaba mis estudios de licenciatura en filosofía, a mediados de la dé-
cada de los años setenta del siglo xx, cuando conocí a Enrique Dussel
Ambrossini en la ciudad de Toluca, quien participó como ponente en
el primer evento sobre filosofía latinoamericana organizado por la Universidad Autónoma del Estado de México (UAEM), junto con Leopoldo Zea, Abelardo Villegas, Ignacio Sosa Álvarez, María Elena Rodríguez y otros destacados latinoamericanistas. Las exposiciones en este suceso tuvieron como saldo avivar mi interés por la filosofía latinoamericana.

La ponencia de Enrique Dussel acerca de la filosofía de la liberación fue eminentemente didáctica al plantear la pertinencia de cuestionar, desde sus fundamentos, la filosofía de los países centrales para sustituirla por una filosofía orientada a coadyuvar a la liberación de los países periféricos.

Más tarde leí su participación en el Primer Coloquio Nacional de Filosofía organizado en Morelia, "La filosofía de la liberación en Argentina, irrupción de una nueva generación filosófica" que apareció en Revisión filosófica, revista de efímera vida del Instituto de Humanidades de la UAEM, donde reseña el origen, compromiso de sus gestores y su razón esclarecedora al suscribir:

Desde que el "yo conquisto" de Cortés se transformó en el ego cogito cartesiano o en la "Willen zur Macht" de Nietzsche, no se ha levantado en nombre del "conquistador", del "pensado" como idea o de la "voluntad impotente" (porque dominado), un pensar crítico metafísico. La filosofía (desde la lógica o la ontología, hasta la estética o la política) desde el Otro, el oprimido, el pobre: el no ser, el bárbaro, la nada de "sentido".

Hay "alguien" más allá de "ser": es real aunque no tenga todavía sentido. ${ }^{1}$

Al realizar mis estudios de posgrado en la Universidad Nacional Autónoma de México asistí a algunas de sus conferencias que en ella dictó, y a las que expuso en los congresos de la Asociación Filosófica de México años después,

${ }^{1}$ Enrique Dussel, "La filosofía de la liberación en Argentina, irrupción de una nueva generación filosófica”, en Revisión filosófica, núm. 1, Toluca, Instituto de Humanidades de la Universidad Autónoma del Estado de México, 1977, pp. 69 y 70. 
donde su postura comprometida, crítica y propositiva me permitió visualizar su praxis liberadora, la cual confirmé cuando participamos en la Comisión Nacional de Homenaje a José Carlos Mariátegui, en el centenario de su nacimiento, a convocatoria de Pablo González Casanova. En esa ruta de compromiso intelectual he sido testigo de su participación en la defensa de la soberanía de nuestros países, la denuncia contra las dictaduras militares, su lucha a favor de los derechos cívicos y en pro de las reivindicaciones étnicas.

Ese conjunto de hechos y los análisis rigurosos que ha producido sobre la realidad para desentrañar los intereses justificadores de la dependencia, el sojuzgamiento y la explotación de los pueblos periféricos, pone de manifiesto la consistencia de sus planteamientos y compromisos, cuya cobertura intelectual la constituye su filosofía de la liberación, que tiene como fuentes la teología de la liberación, la teoría de la dependencia, diversos aportes de las ciencias sociales, el pensamiento de Karl Marx y la filosofía de Emmanuel Levinas.

Con el respaldo de esa concepción filosófica se puede amparar y explicar la praxis liberacionista de Enrique Dussel. Praxis porque sus ideas le han permitido reconocer que la existencia de la injusticia es la principal invocación moral para comprometerse con su superación. La realidad latinoamericana se manifiesta con la dominación, exclusión y explotación. Por eso ha tomado partido por los explotados, entre ellos los indígenas, pero lo ha asumido como filósofo porque para él: "El auténtico filósofo es hombre de su pueblo con su pueblo, pobre junto al pobre $[\ldots]$ y primer profeta del futuro".

Su liberacionismo teológico, filosófico y práctico queda sustentado en su obra teórica como en su excitación a la participación política, en su postura anticapitalista y claro está en la forma como trata el tema del indio, tanto en la explicación de su génesis, en su ubicación como dominado y en la necesidad de mostrar las posibilidades de superación de esa situación, entre otras razones porque: "El rostro pobre, del indio dominado, del mestizo oprimido, del pueblo latinoamericano es el tema de la filosofía latinoamericana".3.

2 Enrique Dussel, Para una ética de la liberación latinoamericana, Buenos Aires, Siglo XxI, 1973, t. II, p. 173.

3 Ibid., p. 164. 
Dentro de la amplia obra de Enrique Dussel existen diversas referencias al indio americano. Las obras dedicadas específicamente al tema son: su tesis doctoral en la década de los años sesenta -El episcopado hispanoamericano (15041620). Institución misionera defensora del indio- y otro texto de los noventa -1492. El encubrimiento del otro (hacia el origen del mito de la modernidad)-, materiales suficientes para internarse en la revisión de su concepción acerca del indio.

\section{CONCEPCIÓN DEL INDIO}

Sus preocupaciones relativas al indígena han sido recurrentes y provienen de sus primeros escritos, según confiesa:

[...] desde nuestras primeras obras, en 1964, vimos la importancia de un replanteo total de la Historia Mundial para descubrir el lugar de América Latina, del "indio" desde el cual comenzamos nuestra interpretación [...] "El Otro" -nuestro maestro Levinas de la década del 60 en París nos sugirió el tema del indio como Otro, en una conversación personal y refiriéndome al "holocausto" indígena-, en su Exterioridad, es el origen de una diferente interpretación de la historia. ${ }^{4}$

Como puede corroborarse, la génesis de sus reflexiones relacionadas con la historicidad del indio provienen de su interés por construir otra historia, la de la periferia, desde ella y por inspiración de uno de sus distinguidos maestros. Por lo cual resulta explicable que en 1966 sustente como tesis doctoral el trabajo intitulado El episcopado hispanoamericano (1504-1620). Institución misionera defensora del indio, en la Universidad de La Sorbona, donde afirma que ya se colocaba " $[. .$.$] desde la perspectiva del indio". 5$

${ }^{4}$ Enrique Dussel, 1492. El encubrimiento del otro (hacia el origen del mito de la modernidad), Santafé de Bogotá, Antropos, 1992, p. 92.

5 Ibid., nota 23, p. 91. 
De modo que su compromiso con los empobrecidos, los excluidos, los de abajo, lo lleva a tratar de "ver" con "sus ojos", ${ }^{6}$ y sustentar que el indio es producto de la invasión y colonización de la modernidad encubridora, que lo ha hecho su víctima. ${ }^{7}$

Con lo cual afirma la necesidad no sólo de interpretar el problema, sino de plantear vías de superación. Así, a lo largo de su obra se observa su compromiso con la liberación de los oprimidos y, obviamente, con el indio, al grado de que sobre él ha desarrollado un enfoque interpretativo particular del cual se desprende una concepción benévola, comprehensiva, pero también rigurosa y crítica. En una visión de conjunto puede apuntarse que su indigenismo incluye tres aspectos relevantes: 1) la justipreciación del periodo prehispánico; 2) la explicación del origen del indio como producto de la modernidad capitalista y, 3) su pensamiento descolonizador. Revisemos cada uno de ellos.

\section{Valoración del periodo prehispánico}

Su apreciación positiva acerca de la época precolombina la sustenta al señalar que diferentes pueblos tuvieron autonomía, esplendor social y evidentes manifestaciones culturales al reconocer que su " [...] visión mitológico-ritual suponía un altísimo grado de racionalización. Los mitos suponen una racionalidad de alto grado de criticidad, supone ya una cierta ilustración". ${ }^{8}$

Tal interpretación no le impide radiografiar la diversidad cultural como de los desarrollos socioeconómicos de las culturas de entonces al ubicarlas en distintos grados: 1) los clanes y tribus de pescadores, cazadores y recolectores; 2) los plantadores con aldeas de clanes, tribus y confederaciones y, 3) las sociedades urbanas mesoamericanas y andinas.

${ }^{6}$ Enrique Dussel, El encubrimiento del indio: 1492. Hacia el origen del mito de la modernidad, México, Cambio xxi/Colegio Nacional de Ciencias Políticas y Administración Pública, 1994, p. 121. Esta obra es en realidad otra edición, con título mínimamente modificado, de la citada en la nota 4 .

7 Ibid., p. 185.

${ }^{8}$ Ibid., p. 126.

${ }^{9}$ Cfr. Ibid., pp. 139-141. 
Pese a sus variados grados de desenvolvimiento destaca como virtudes, en general, sus sistemas de total reciprocidad, el profundo valor y respeto de la palabra, las distintas manifestaciones de racionalidad, la veneración e interacción con la tierra, la sistematización de saberes de alto grado de abstracción como su pensamiento protofilosófico realizada por los tlamatinime y los amautas, sus sistemas educativos muy bien organizados, diferenciados y jerarquizados e incluso la justificación del heroísmo de sus resistencias ante las invasiones. Todo eso y más se palpa al repasar muchas de las páginas de algunas de sus obras.

\section{El indio producto de la modernidad capitalista}

Pero ese desarrollo prehispánico quedó "en-cubierto", como primer momento del origen del indio, a partir de los tiempos del "descubrimiento" de América por los europeos, por lo que desde 1492 diacrónicamente se proyectó sobre todo el continente la barbarización ${ }^{10}$ que preparó la segunda fase de la génesis del indio, la conquista, y consolidó la tercera, la colonial, cuyos rasgos persisten. La constitución del indio como resultado de este triple proceso histórico puede explicitarse con base en su obra, en seis puntos.

1. Génesis del indio. Enrique Dussel identifica el arribo de los europeos al Nuevo Mundo como el momento inicial de la constitución del indio como realidad histórica. Lo hace al especificar las fases de la teología de la liberación: "[... A Al comienzo, y por el ahora tan celebrado 'descubrimiento' que en realidad fue conquista, violencia y muerte de los amerindios, los pobres fueron los indigenas o primitivos habitantes americanos [...]", ${ }^{11}$ de modo que el indio advino como consecuencia del descubrimiento, pues la violencia que engendró ha persistido hasta nuestros días. En efecto, el indio es producto del capitalismo expansionista desde sus orígenes, al mantenerlo como reserva de mano de obra barata y consumidor real o potencial.

${ }^{10}$ Ibid., pp. 146 y 147.

${ }^{11}$ Enrique Dussel, Teología de la liberación. Un panorama de su desarrollo, México, Potrillos Editores, 1995 , pp. 5 y 6. 
Pero la génesis del indio tuvo implicaciones de trascendencia histórica para el desarrollo del mundo occidental, que lo hizo posible, pues en la perspectiva de Enrique Dussel dio origen a la "Modernidad" al plantear:

En 1492, según nuestra tesis central, es la fecha del "nacimiento" de la Modernidad; aunque su gestación - como feto- lleva un tiempo de crecimiento intrauterino. La modernidad se originó en las ciudades europeas medievales, libres, centros de enorme creatividad. Pero "nació" cuando Europa pudo confrontarse con "el Otro" [... y controlarlo, vencerlo, violentarlo; cuando pudo definirse como un "ego" descubridor, conquistador, colonizador de la Alteridad constitutiva de la misma Modernidad. De todas maneras, ese Otro no fue "descubierto" como Otro, sino que fue "en-cubierto" como "lo Mismo" [... ]. De manera que el 1492 será el momento del "nacimiento" de la Modernidad como concepto correcto, el "origen" de un "mito" de violencia sacrificial muy particular, y, al mismo tiempo, un proceso de "en-cubrimiento" de lo no-europeo. ${ }^{12}$

Con lo cual prueba el advenimiento de América como la " [... ] primera 'periferia' de la Europa Moderna", ${ }^{13}$ como su alteridad, porque desde entonces "[...] ha constituido a las otras culturas, mundos, personas como ob-jeto: como lo 'arrojado' (-jacere) 'ante' (ob-) sus ojos [...]”. ${ }^{14}$

La consolidación de la constitución del indio continuó con la conquista, mediante las acciones genocidas y devastadoras sobre el mundo prehispánico, situación que se ha encubierto de múltiples maneras, llegando a hechos inverosímiles, dice Enrique Dussel, como la "[... ] gigantesca inversión: la víctima inocente es transformada en culpable, el victimario culpable es considerado inocente [...]", ${ }^{15}$ al imponerse al manto del olvido, la barbarización, la modernización.

Así explica que la existencia del "[...] primer protagonista de la historia latinoamericana [...] son los indios [...] cuya historia posterior dura ya 500 años" ${ }^{16}$ Durante estas cinco centurias de subsistencia ha vivenciado diversidad

${ }^{12}$ Dussel, 1492. El encubrimiento del otro..., p. 182.

${ }^{13}$ Ibid., p. 20

${ }^{14}$ Ibid., p. 53.

${ }^{15}$ Ibid., p. 106.

${ }^{16}$ Dussel, El encubrimiento del indio..., p. 182. 
de situaciones que oscilan entre su constitución como dominado y su resistencia persistente. La explicación de ambos aspectos se encuentra en distintas obras de nuestro autor por lo que resulta pertinente continuar con su análisis.

2. Inicio de la dominación militar. El siguiente acto de conquista, de dominio, en estricto sentido, lo constituyó la acción de fuerza de los europeos, que Enrique Dussel explica como la práctica militar en una relación de persona-persona, del control de los cuerpos, de las personas, de los pueblos, de los "indios", como "praxis" de la dominación, por lo que concibe al "conquistador", el primer hombre moderno activo, como el sujeto que impone su "individualidad" violenta a otras personas, al Otro. ${ }^{17}$ De modo que el establecimiento de esta relación conquistador-conquistado redujo al aborigen del Nuevo Mundo a indio, sobre quien se formó la imagen de primitivo, rústico, inferior, incapaz, negación del Otro en otro.

La violencia con la que se procedió en el acto de la conquista militar fue justificada irracionalmente con careta racional. Así recuerda Dussel que los conquistadores leían, en idioma ajeno e incomprensible, a los indígenas un texto de "requerimiento" antes de las batallas mediante el cual les proponían la conversión a la religión cristiano-europea con el propósito de evitarles el dolor de la derrota. ${ }^{18}$

3. Explotación de la fuerza de trabajo amerindia. El proceso de conquista no concluyó con el sometimiento militar, sino se acentuó con la explotación de la fuerza de trabajo de los indios, que desde el siglo xvi hasta la fecha se ha efectuado en las más diversas instituciones económicas del capitalismo realmente existente: la esclavitud, la encomienda, la minería, la hacienda, el obraje o los distintos tipos de industria. Al respecto Dussel apunta:

Brutal y violentamente incorporado primero a la "encomienda" —explotación gratuita del trabajo indígena-, posteriormente a los "repartimientos", sean agrícolas o mineros (la "mita andina"), para por último recibir salarios de hambre en las "haciendas", el indio deberá recomponer totalmente su existencia para sobrevivir en una inhumana opresión: las primeras víctimas de la modernidad —el primer "holocausto" moderno lo llama Russel Thorntont- ${ }^{19}$.

\footnotetext{
${ }^{17}$ Dussel, 1492. El encubrimiento del otro..., pp. 55 y 56

${ }^{18}$ Ibid., p. 180.

${ }^{19}$ Dussel, El encubrimiento del indio..., p. 182.
} 
Al grado de que la explotación económica en el tiempo de la acumulación originaria del capitalismo mercantil, la corporalidad india devino, primero, oro y plata, valor muerto de la objetivación del "trabajo vivo"; ${ }^{20}$ así fue subsumido en la totalidad del nuevo sistema económico como mano de obra gratis o barata ${ }^{21}$ y con el paso de los años su explotación lo ha reconvertido en obrero agrícola, de la construcción, de la industria o de los servicios.

4. Destrucción de la tradición cultural prehispánica. El medio por el cual fue consolidada la dominación europea sobre el indio, lo constituyó la destrucción de las principales manifestaciones culturales de las sociedades mesoamericanas y andinas que habían alcanzado elevados niveles de creatividad intelectual.

Para sustentar tal apreciación, basta recuperar referencias de la obra de Enrique Dussel porque aclaran las formas de vida cotidiana conquistadora-europea con que "colonizaron" el mundo de la vida de los amerindios, ${ }^{22}$ pues comenzó con la destrucción de sus principales elementos culturales, ya que tanto las producciones de aztecas como de incas fueron concebidas como inferiores, bárbaras, rústicas, porque no llegaron al uso de la escritura ni al cultivo del saber filosófico $^{23}$ semejante a Occidente.

De tal forma que les fueron destruidas las condiciones para que persistiera el cultivo de los desarrollos de sus saberes racionales, de sus cosmovisiones y prácticas religiosas, y por consecuencia la creatividad intelectual de los tlamatinime y amautas desaparecieron. Al respecto clarifica Enrique Dussel: "Reducidos en número, extirpadas las élites de las civilizaciones indígenas, el pueblo de los pobres sobrevivió sin poder ya revivir el esplendor del pasado". ${ }^{24}$

5. Imposición de elementos culturales occidentales. La transformación del aborigen americano en indio tuvo como principal refuerzo el cambio de su mentalidad, a través de la imposición de elementos culturales que le eran ajenos como

${ }^{20}$ Dussel, 1492. El encubrimiento del otro..., p. 74.

${ }^{21}$ Ibid., p. 75.

${ }^{22}$ Ibid., p. 71.

${ }^{23}$ Ibid., p. 85.

${ }^{24}$ Dussel, El encubrimiento del indio..., p. 183. 
el indio, la religión y diversidad de prácticas económicas, políticas y sociales. Enrique Dussel identifica este proceso de conquista cultural como la larga historia de colonización, que por cierto aún padece. Este proceso tuvo como resultado hacer al aborigen indio, esto es parte de la modernidad-capitalista, su "otra-cara", la cara explotada, dominada, encubierta, oprimida. ${ }^{25}$ Incluso con base en sus lúcidos análisis sobre la religión católica ha concluido que la llegada de los primeros misioneros, en 1524, dio inicio a la conquista espiritual, ${ }^{26}$ toda vez que la acción evangelizadora culminó principalmente en ideología justificadora de la dominación. Así revela: " [... El El sentido encubierto era que en realidad los europeos habían dominado al indio reduciéndolo a la más horrible servidumbre. La muerte, el robo, la tortura (que era el fruto real de la praxis conquistadora) quedaban cubiertas por la interpretación ideológica: la evangelización". ${ }^{27}$

El efecto de la nueva cultura que se le impuso radicó en cubrir el vacío de la destrucción de la tradición intelectual forjada por sus ancestros prehispánicos, y desempeñar los papeles ideológicos para mantener su nueva realidad de dominado.

Obviamente, ese proceso de imposición de la nueva cultura, la de la modernidad capitalista, no terminó con la conquista en el siglo xvI, puesto que persistió durante la época colonial y la vida republicana al grado que "[...] el segundo golpe fatal lo recibirán del liberalismo del siglo xIx que pretendiendo imponer una concepción de la vida 'ciudadana' abstracta, burguesa, individualista, comenzó a imponer la propiedad privada del campo, y luchó contra la 'comunidad' como modo de vida, lo que hizo aún más difícil que antes la existencia del indio". ${ }^{28}$

Precisamente, con el advenimiento de los regímenes republicanos en América Latina la condición del indio no mejoró, pues sigue siendo objeto de imposición de más elementos de la cultura moderna: la nueva religión, por ejemplo, es el nacionalismo con la historia de bronce que le inculturan los gobiernos de los países donde habita lo cual, de paso, le ha venido cercenando el saber de sus antepasados.

\footnotetext{
${ }^{25}$ Dussel, 1492. El encubrimiento del otro..., p. 79

${ }^{26} \mathrm{Ibid}$., p. 82.

${ }^{27}$ Dussel, Teología de la liberación..., p. 15.

${ }^{28}$ Dussel, El encubrimiento del indio..., p. 183.
} 
6. Conformación de su situación colonial. La creación del indio como producto de la modernidad capitalista trajo aparejado el intento de intimación de una ideología no sólo justificadora de la dominación, sino orientada a internalizarle resignación y aceptación incuestionable de ella. Este develamiento que realiza Enrique Dussel lo expone de diversas formas al recoger la propia tradición pesimista de las sociedades prehispánicas con la admisión de la situación trágica a que se les redujo, ${ }^{29}$ de conformarse con ella.

A ese tipo de justificación colonialista se deben añadir los distintos mecanismos usados para forjar en el indio la autoimagen de analfabeto, indolente, pasivo, resignado, sumiso, con el propósito de prevenir cualquier manifestación contraria a su dominación. También es pertinente apuntar que su herencia y fuerza han sido usadas por otros sectores sociales para su beneficio, como los casos de criollos y mestizos al expropiarles sus símbolos como la virgen de Guadalupe, ${ }^{30}$ el pasado de sus ancestros prehispánicos o como carne de cañón.

\section{Pensamiento descolonizador}

La praxis liberadora de Enrique Dussel sintetiza también sus estudios y reflexiones sobre el indio con base en el carácter comprometido mediante el cual contextualiza su situación. El carácter liberador se respalda en el meticuloso análisis como procede, pues sustenta la constante y larga resistencia del indio a la conquista; destaca su espíritu de rebeldía; plantea la necesidad de descolonizar su situación; propone incorporarlo como parte de los sujetos de la transformación social de los oprimidos, y esboza la pertinencia de hacerlo partícipe de la construcción de un nuevo proyecto societario. Expliquemos cada uno de esos aspectos que reflejan su pensamiento descolonizador.

1. Resistencia a la conquista. El enfoque liberador con el que procede Enrique Dussel lo pone de manifiesto en sus exposiciones relativas al estado de opresión y resistencia de los indios, como lo prueba su tesis doctoral y lo reitera en otros textos. El cúmulo de señalamientos relacionados con la permanente ac-

${ }^{29}$ Dussel, 1492. El encubrimiento del otro..., p. 194.

${ }^{30}$ Ibid., p. 193. 
titud de entereza de los indios lo ubica en las respuestas de éstos a las situaciones de dominación a las que se les han reducido.

Los datos con los cuales prueba sus planteamientos los obtiene de los testimonios que localiza en la obra de los cronistas ${ }^{31}$ y los estudiosos de la historia de los indígenas. Por ejemplo relata que:

Se podría seguir paso a paso la "resistencia" ante la "invasión", pero sólo indicaremos algunos de los rasgos de la misma. En la Hispañola (Santo Domingo...), había cinco pequeños reinos bajo el mando de los caciques Goarionex, Guacanagarí (el traidor de su pueblo y "amigo" de Colón), Canoabo, Behechio (hermano de la reina Anacaona famosa por su valentía y belleza) y Catubanamá. Fue Canoabo, en Cibao, el que resistió al robo de las mujeres de su pueblo perpetrada por los españoles dejados por Colón en el fuerte de Navidad. Estos robaban, violaban, mataban indios. El cacique se dirigió al fuerte y ajustició a los invasores. Fue el comienzo de la resistencia en el continente. ${ }^{32}$

De forma tal que al identificar el inicio de la resistencia india continúa inventariando sus persistentes acciones contra el proceso de conquista, que no termina. Cita los ejemplos de Xicoténcatl en Tlaxcala, Cacama en Texcoco, Cuauhtémoc en Tenochtitlán; la lucha de liberación de los mayas, que sigue hasta nuestros días, al destacar como forjadores de esa tradición de resistencia a Tecum Uman, Jacinto Canek, Lempira; los casos de los caciques Guaicaipuro y Yaracuy en Colombia; y la de los incas encabezados por Rumiñahui, Quizquiz, Calichima, Manco Capac, Tupac Amaru; el caso heroico de los araucanos, en particular las luchas encabezadas por Lautaro y Caupolican..$^{33}$

Con lo cual, me parece, se corrobora su análisis clarificador y desalienante, al concluir que la resistencia indígena es parte de su existencia a lo largo de los cinco siglos, pues nunca ha aceptado como natural ni resignadamente la situación colonial que lo engendró y padece.

2. Rebeldía como base de la descolonización. El cúmulo de luchas de resistencia del indio constituye una manifestación incuestionable de su actitud de

${ }^{31}$ Dussel, El encubrimiento del indio..., p. 188.

${ }_{32}$ Dussel, 1492. El encubrimiento del otro..., p. 189.

${ }^{33}$ Cfr. Ibid., pp. 188-192. 
rebeldía, orientada a trascender el colonialismo que padece. Dussel argumenta persistentemente la necesidad de coadyuvar a su desalienación de las falacias esgrimidas por los ideólogos de la conquista y la modernidad capitalista, que pretenden reducirlo a mero accidente de la historia.

Responsabilizarlo de su situación de dominado sin considerar que tuvo la posibilidad de conversión pacífica, más bien evidencia el propósito de castrarle toda oportunidad de superar su condición opresiva, porque se ha pretendido presentar su rebeldía como osadía a su integración a la modernidad capitalista, ${ }^{34}$ argumento producto del poder emanado de la conquista, que imposibilita todo diálogo entre "la razón del Otro" y el discurso de la modernidad al imponerse mediante el adoctrinamiento. ${ }^{35}$ Debido a esa ruptura de argumentos, Dussel reconoce que la actitud de rebeldía contará con bases para mantenerla y concretarla recurrentemente por la inspiración de sus sabios prehispánicos, porque nunca aceptaron la "verdad" de los dominadores.

Dicha rebeldía permanece como lo prueba, por ejemplo, el dato de que el 11 de febrero de 1988, la Asociación Indígena Salvadoreña, en su I Encuentro Espiritual y Cultural, repudió la "invasión extranjera" de América y declaró: "[...] un alto al genocidio y etnocidio de subpueblos y culturas y el rechazo a la celebración de los 500 años de la invasión extranjera" ${ }^{36}$

Para comprender mejor esta posición de Enrique Dussel es pertinente señalar la diferencia que hace de las concepciones relativas a la modernidad cuando escribe:

La Modernidad en su núcleo racional es emancipación de la humanidad del estado de inmadurez, cultural, civilizatoria. Pero como mito, en el horizonte mundial, inmola a los hombres y mujeres del mundo periférico, colonial (que los amerindios fueron los primeros en sufrir), como víctimas explotadas, cuya victimación es encubierta con el argumento del sacrificio o costo de la modernización. Este mito irracional es el horizonte que debe trascender el acto de liberación $[\ldots] .^{37}$

${ }^{34} \mathrm{Ibid}$. p. 105

${ }^{35} \mathrm{Ibid}$. p. 198

${ }^{36}$ Dussel, El encubrimiento del indio..., p. 183.

${ }^{37}$ Dussel, 1492. El encubrimiento del otro..., p. 209. 
De modo que la rebeldía indígena tendrá un horizonte claro si se hace eco y sistematiza su propio pensamiento, como en varias ocasiones lo recoge y busca potenciarlo al sumarlo a los "rostros" de campesinos y negros.

3. Identificación del indio como sujeto de transformación social. Al compartir la opinión de que parte de la labor intelectual de Enrique Dussel estriba en intentar reconstruir el sujeto histórico de las transformaciones en América Latina, ${ }^{38}$ puede fundamentarse que mediante la filosofía de la liberación del oprimido pretende rescatar al indio como parte activa del proceso de emancipación. Sus propias palabras lo confirman:

[... ] la "Filosofía de la Liberación", que parte desde la Alteridad, desde el "compelido" o el "excluido" (la cultura dominada y explotada), de lo concreto-histórico [...] trata de mostrar esas condiciones de posibilidad de dialogar, desde la afirmación de la Alteridad, y, al mismo tiempo, desde la negatividad, desde su imposibilidad empírica concreta, al menos como punto de partida, de que "el-Otro-excluido" y "dominado" pueda efectivamente intervenir $[. . .]^{39}$

Esta posición ética obviamente la extiende a todo oprimido, no sólo al indio, aunque de él esclarezca diversos aspectos de su tradición como sujeto histórico, como la anotación de que sus ancestros prehispánicos fueron los primeros en descubrir el continente americano a través de sus experiencias telúricas, ciertamente regionales, y vivenciales en general, mucho antes que el "descubrimiento europeo" ${ }^{\text {"40 }}$ y porque desde la realidad de la dominación surge la posibilidad de la liberación.

Por ese conjunto de planteamientos, su obra tiene el cometido de intentar restaurar el diálogo intercultural que fue interrumpido al inicio de la conquista, con lo cual busca coadyuvar a trastocar la situación colonial del indio al convertirlo en sujeto de la historia, con el cometido de reconocerlo partícipe de la lucha por su liberación.

4. Pertinencia de nuevo proyecto societario. La florida y erudita exposición de Enrique Dussel acerca de la racionalidad de los sabios de la época prehispánica,

${ }^{38}$ Renato Rodríguez, "Prólogo", en ibid., p. 9.

${ }^{39}$ Dussel, 1492. El encubrimiento del otro..., p. 13.

${ }^{40}$ Ibid., nota 6, p. 120. 
no debe percibirse ni como una idealización sin más de ese periodo ni como el proyecto de sociedad al cual debe retornarse, en principio porque la saña con la cual los invasores destruyeron las bases culturales no ha tenido parangón en la historia de la humanidad, con lo que resulta imposible su resurgimiento.

No, el proyecto societario por el cual pugna Enrique Dussel es el de la "Transmodernidad", ${ }^{41}$ el cual naturalmente no niega la razón, sino la irracionalidad de la violencia del mito moderno -el forjador de la existencia del indio como dominado-, niega la irracionalidad posmoderna, pero afirma, en cambio, la "razón del Otro" para edificar una mundialización transmoderna. ${ }^{42}$

Y, claro, dentro de ese proyecto plantea la indispensable integración de la tradición libertaria y las manifestaciones preclaras de la cultura de todos los oprimidos, entre ellos los indígenas, por lo que apunta como contenido lo " $[\ldots$. . racional, como reconstructivo del mito; práctico-político, como acción que supera el capitalismo y la modernidad en un tipo transmoderno de civilización ecológica, de democracia popular y de justicia económica". ${ }^{3}$

Por lo cual este proyecto liberador, no sólo asuntivo, concreta la superación de la modernidad, la racionalidad ampliada en la que la razón del Otro tendrá cabida, la comunidad de comunicación en la que todos los humanos participen como iguales y con profundo respeto a su alteridad. ${ }^{44}$ Así se puede concluir que el pensamiento descolonizador tiene como fin forjar los pilares para construir una nueva humanidad.

Con base en dichos planteamientos, Enrique Dussel Ambrossini puede ser ubicado como uno de los pensadores que mayores contribuciones ha venido realizando al esclarecimiento del problema del indio y, por su praxis liberadora, resulta encomiable reconocerlo como uno de los más connotados representantes del marxismo indigenista, en particular, del marxismo descolonizador ${ }^{45}$

${ }^{41}$ Ibid., p. 11 .

${ }^{42}$ Ibid., p. 34.

${ }^{43}$ Ibid., p. 209

${ }^{44}$ Dussel, El encubrimiento del indio..., p. 202.

${ }^{45}$ Alberto Saladino García, "Teoría revolucionaria y cuestión indígena”, en Dialéctica, Nueva Época, núm. 28, Puebla, invierno, 1995/1996, pp. 102-108. 
Así, el sello distintivo de su pensamiento estriba en exhibir que la dependencia que padecen las etnias no sólo es la de nuestros países frente a los países centrales, sino la que padecen los sectores dominantes de los países donde subsisten, por lo cual su realidad histórica y su vida presente es de un sufrimiento de doble colonialismo: el externo - que creó al indio-y el interno - que reproduce sus condiciones de postración.

Como consecuencia, el quehacer filosófico de Enrique Dussel enfrenta, de manera directa, la dependencia y el colonialismo, tanto externo como interno, pues lo percibe como una forma de saber generado desde fuera del sistema de los países centrales, que parte de la conciencia del oprimido como alteridad. De modo que toda su labor intelectual puede identificarse como praxis orientada a forjar el advenimiento de la real liberación humana.

Recibido: 19 de abril, 2010. Aceptado: 17 de mayo, 2010.

\section{BiBLIOgRAFÍA}

Dussel, EnRiQue, Para una ética de la liberación latinoamericana, Buenos Aires, Siglo XXI, t. II, 1973.

, "La filosofía de la liberación en Argentina, irrupción de una nueva generación filosófica", en Revisión Filosófica, núm. 1, Toluca, Instituto de Humanidades, Universidad Autónoma del Estado de México, 1977. , 1492. El encubrimiento del otro (bacia el origen del mito de la modernidad), Santafé de Bogotá, Antropos, 1992.

, El encubrimiento del indio: 1492. Hacia el origen del mito de la modernidad, México, Cambio xxi/Colegio Nacional de Ciencias Políticas y Administración Pública, 1994. 
,Teología de la liberación. Un panorama de su desarrollo, México, Potrillos Editores, 1995.

Saladino García, Alberto, "Teoría revolucionaria y cuestión indígena", en Dialéctica, Nueva Época, núm. 28, Puebla, invierno, 1995/1996. 